\title{
A NEW SDM CLASSIFIER USING JACCARD MINING PROCEDURE CASE STUDY: RHEUMATIC FEVER DATA
}

\author{
${ }^{1}$ M.E. Abd El-Monsef, ${ }^{2}$ E. A. Rady, ${ }^{3}$ A. M. Kozea, \\ ${ }^{4}$ W. A. Hassanein, ${ }^{5}$ S. Abd El-Badie \\ $\mathbf{1 , 3 , 4 , 5}$ Mathematics Department, Faculty of Science, Tanta University, Tanta, Egypt \\ ${ }^{2}$ Institute of Statistical Studies \& Research (ISSR), Cairo University, Cairo, Egypt
}

\begin{abstract}
In this paper, a new Statistical Data Mining (SDM) technique is proposed using Jaccard Mining Procedure $(J M P)$ contributing a novel classifier \& predictor by applying very effective stages on the training data depending on Jaccard $(\mathrm{J})$ distance matrix Linked with the Gini Index Measure as precision measure for initiating a new classifier and a new predictor, The proposed SDM technique using JMP is applied and examined on a Rheumatic Fever Data to demonstrate its applicability.
\end{abstract}

\section{KEYWORDS}

Jaccard, Classifier, Mining, Gini, Rheumatic Fever, Data

\section{INTRODUCTION}

Classification $[3,4,5,7]$, is a data mining function that assigns items in a collection to target categories or classes. The goal of classification is to accurately predict the target class for each case in the data. For example, a classification model could be used to identify loan applicants as low, medium, or high credit risks.

A classification task begins with a data set in which the class assignments are known. For example, a classification model that predicts credit risk could be developed based on observed data for many loan applicants over a period of time. In addition to the historical credit rating, the data might track employment history, home ownership or rental, years of residence, number and type of investments, and so on. Credit rating would be the target, the other attributes would be the predictors, and the data for each customer would constitute a case.

The simplest type of classification problem is binary classification. In binary classification, the target attribute has only two possible values: for example, high credit rating or low credit rating. Multiclass targets have more than two values: for example, low, medium, high, or unknown credit rating.

Different classification algorithms [7] use different techniques for finding relationships. These relationships are summarized in a model, which can then be applied to a different data set in which the class assignments are unknown. Classification has many applications in customer segmentation, business modeling, marketing, credit analysis, and biomedical and drug response modeling.

DOI: $10.5121 / \mathrm{ijbb} .2014 .4101$ 
There is a difference between the definition of the algorithm and the flowchart [6], an algorithm is just a detailed sequence of simple steps that are needed to solve a problem, from the other side; a flowchart is a graphical representation of an algorithm. So we will introduce the flowchart and the algorithm o our paper.

The sequence of this paper is organized as follows; Section 2 represents the materials \& methods which are subtitled into, Rheumatic Fever Data Characteristics, Jaccard distance (J) \& Gini Index. Section 3 presents the JMP algorithm, JMP flow chart and JMP systematic structure stages using Jaccard distance, Jaccard classes, Jaccard classifiers linking the previous stages with the Gini Index as precision measures for initiating a new classifier and concluding a new predictor. Finally, the conclusion and the future work are introduced in Section 4.

\section{JMP MATERIALS \& METHODS}

\subsection{Rheumatic Fever Data: Characteristic}

No doubt that the Rheumatic Fever is a very common disease [2] and it has many symptoms differs from patient to another though the diagnosis is the same. So, we obtained the following example on seven rheumatic fever patients from Tanta University Hospital, Egypt. All patients are between 9-12 years old with history of Arthritis began from age 3-5 years. This disease has many symptoms and it is usually started in young age and still with the patient along his life. Table (1) introduced seven patients characterized by 8 symptoms (attributes) [1] using them to decide the diagnosis for each patient (decision attribute). Table (2) introduces the training coded data. Where, $\{\mathrm{S}, \mathrm{F}, \mathrm{A}, \mathrm{R}, \mathrm{K}, \mathrm{E}, \mathrm{P}, \mathrm{H}\}$ are the conditional attributes, $\{\mathrm{P} 1, \mathrm{P} 2, \mathrm{P} 3, \ldots, \mathrm{P} 7\}$ in Table (2) are the Rheumatic Fever training data objects and the diagnosis attribute (D) as the decision attribute.

\begin{tabular}{|c|c|c|c|}
\hline At. Name & At. Symbol & At. Code & At. Description \\
\hline \multirow{2}{*}{ Sex } & \multirow{2}{*}{$S$} & 1 & Male \\
\hline & & 2 & Female \\
\hline \multirow{2}{*}{ Pharyngitis } & \multirow{2}{*}{$\mathrm{F}$} & 1 & No \\
\hline & & 2 & Yes \\
\hline \multirow{2}{*}{ Arthritis } & \multirow{2}{*}{ A } & 1 & No Arthritis \\
\hline & & 2 & Arthritis \\
\hline \multirow{2}{*}{ Carditis } & \multirow{2}{*}{$\mathrm{R}$} & 1 & Not Affected \\
\hline & & 2 & Affected \\
\hline \multirow{2}{*}{ Chorea } & \multirow{2}{*}{ K } & 1 & No \\
\hline & & 2 & Yes \\
\hline \multirow{2}{*}{ ESR } & \multirow{2}{*}{$\mathrm{E}$} & 1 & Normal \\
\hline & & 2 & High \\
\hline \multirow{2}{*}{$\begin{array}{l}\text { Abdominal } \\
\text { Pain }\end{array}$} & \multirow{2}{*}{$\mathrm{P}$} & 1 & No \\
\hline & & 2 & Yes \\
\hline \multirow{2}{*}{ Headache } & \multirow{2}{*}{$\mathrm{H}$} & 1 & No \\
\hline & & 2 & Yes \\
\hline \multirow{3}{*}{ Diagnosis } & \multirow{3}{*}{$\mathrm{D}$} & 1 & Rheumatic Arthritis \\
\hline & & 2 & Rheumatic Carditis \\
\hline & & 3 & Rheumatic Arthritis \&Carditis \\
\hline
\end{tabular}

Table (1): Rheumatic Fever Data Description 


\begin{tabular}{|c|c|c|c|c|c|c|c|c|c|}
\hline & $\mathbf{S}$ & $\mathbf{F}$ & $\mathbf{A}$ & $\mathbf{R}$ & $\mathbf{K}$ & $\mathbf{E}$ & $\mathbf{P}$ & $\mathbf{H}$ & $\mathbf{D}$ \\
\hline $\mathbf{P}_{\mathbf{1}}$ & 2 & 2 & 2 & 2 & 2 & 1 & 1 & 1 & 3 \\
\hline $\mathbf{P}_{\mathbf{2}}$ & 1 & 2 & 2 & 2 & 2 & 2 & 1 & 2 & 3 \\
\hline $\mathbf{P}_{\mathbf{3}}$ & 2 & 2 & 2 & 2 & 1 & 1 & 1 & 1 & 3 \\
\hline $\mathbf{P}_{\mathbf{4}}$ & 1 & 2 & 2 & 1 & 1 & 1 & 1 & 1 & 1 \\
\hline $\mathbf{P}_{\mathbf{5}}$ & 1 & 1 & 1 & 2 & 1 & 1 & 2 & 1 & 2 \\
\hline $\mathbf{P}_{\mathbf{6}}$ & 2 & 2 & 2 & 2 & 1 & 2 & 1 & 1 & 3 \\
\hline $\mathbf{P}_{\mathbf{7}}$ & 1 & 2 & 2 & 2 & 1 & 1 & 1 & 2 & 3 \\
\hline
\end{tabular}

Table (2): Coded Rheumatic Fever Data

\subsection{Jaccard Distance $\left(\mathrm{J}_{\text {Dist. }}\right)$}

It is very important to note that, Jaccard coefficient is a measure for similarity between two variables and Jaccard distance [3] is a measure of dissimilarity and both of them are measurement of asymmetric information on binary and non-binary variables. The definition of the Jaccard similarity coefficient and the Jaccard distance are as follows;

Def.1: Jaccard similarity between binary variables A and B

$$
\mathrm{J}_{\text {Sim }}(\mathbf{A}, \mathbf{B})=\frac{\mathbf{P}(\mathbf{A} \cap B)}{\mathbf{P}(\mathbf{A} \cup \mathbf{B})}
$$

Def.2: Jaccard Distance between binary variables A and B

$$
\mathbf{J}_{\text {Dist. }}(\mathbf{A}, \mathbf{B})=\mathbf{1}-\mathbf{J}_{\text {Sim }}(\mathbf{A}, \mathbf{B})
$$

\section{Example: (Jaccard Distance Calculations)}

\begin{tabular}{|l|l|l|}
\hline & $\mathbf{S}$ & $\mathbf{F}$ \\
\hline $\mathbf{P}_{\mathbf{1}}$ & 2 & 2 \\
\hline $\mathbf{P}_{\mathbf{2}}$ & 1 & 2 \\
\hline $\mathbf{P}_{\mathbf{3}}$ & 2 & 2 \\
\hline $\mathbf{P}_{\mathbf{4}}$ & 1 & 2 \\
\hline $\mathbf{P}_{\mathbf{5}}$ & 1 & 1 \\
\hline $\mathbf{P}_{\mathbf{6}}$ & 2 & 2 \\
\hline $\mathbf{P}_{\mathbf{7}}$ & 1 & 2 \\
\hline
\end{tabular}

$\mathrm{J}_{\mathrm{Sim}}(\mathrm{S}, \mathrm{F})=4 / 7 \rightarrow \mathrm{J}_{\text {Dist }}(\mathrm{S}, \mathrm{F})=3 / 7=0.43$

Similarly for the entire conditional attributes as in Table (3) 


\subsection{Gini Index}

The Gini index [3], measures the impurity of the target attribute, so we will use it as a precision method in our paper. The Gini index of a data partition or set of training tuples, as

$$
\operatorname{Gini}(\mathbf{A})=1-\sum_{\mathbf{j}=1}^{\mathbf{n}} \mathbf{P}_{\mathbf{j}}^{2}
$$

Eq. (3)

Where $p_{j}$ is the relative frequency of class $\mathrm{j}$ in $\mathrm{A}$.

The Gini index considers a binary split for each attribute. Let's first consider the case where B is a discrete-valued attribute having $\mathrm{v}$ distinct values, $\{\mathrm{b} 1, \mathrm{~b} 2 \ldots \mathrm{bv}\}$, occurring in $\mathrm{A}$. When considering a binary split, we compute a weighted sum of the impurity of each resulting partition. For example, if a binary split on B partitions the attribute A into A1 and A2, the Gini index of the attribute A given that partitioning is,

$$
\operatorname{Gini}_{B}(A)=\frac{\left|A_{1}\right|}{|A|} \operatorname{Gini}\left(A_{1}\right)+\frac{\left|A_{2}\right|}{|A|} \operatorname{Gini}\left(A_{2}\right)
$$

In General the Gini of the split formula is,

$$
\operatorname{Gini}(A)=1-\sum_{j}[P(j / A)]^{2}
$$

The most important characteristics of the Gini Index that,

1) It varies between 0 and 1 .

2) If Gini $=0 \rightarrow$ Complete Equality

3) If Gini $=1 \rightarrow$ Complete Inequality

4) If $0<$ Gini $<1 \rightarrow$ the higher the Gini index, the greater the inequality.

5) Biased to multivalued attributes.

6) Has difficulty when number of classes is large.

7) Tends to favor tests that result in equal-sized partitions and purity in both partitions.

\section{Example: (Gini Index Calculations)}

Calculating the Gini of the diagnosis attribute (D) using Eq. (3),

$\operatorname{GINI}(D)=1-\left[(1 / 7)^{2}+(1 / 7)^{2}+(5 / 7)^{2}\right]=0.45$

\& calculating the Gini of the attribute (S) using Eq. (4),

Similarly for the entire conditional attributes as in Table (6).

$$
\begin{aligned}
& \operatorname{GINI}\left(s_{1}\right)=1-\left[(1 / 4)^{2}+(1 / 4)^{2}+(2 / 4)^{2}\right]=0.625 \\
& \& \operatorname{GINI}\left(s_{2}\right)=1-\left[(0 / 3)^{2}+(0 / 3)^{2}+(3 / 3)^{2}\right]=0 \\
& \rightarrow \operatorname{GINI}(S)=[(4 / 7)(0.6)+(3 / 7)(0)]=0.35
\end{aligned}
$$




\section{JMP Systematic Structure Stages}

\subsection{JMP Algorithm}

In this section the JMP algorithm steps will be introduced in the following.

\section{Input}

$$
\text { Training Data }=(U, A \cup D)
$$

\section{Processing}

1. $J \leftarrow\{\}$

2. Do

3. $J$ Classes $\leftarrow J$

4. J Classsifiers $\leftarrow J$ Classes

5. Gini Index $\leftarrow\{\}$

5. J Classsifiers $\leftarrow$ Gini Index

6. Gini Index Averages $\leftarrow$ Gini Index

7. Best JMP Classifier $\leftarrow$ Gini Index Averages

\section{Output}

Best JMP Classifier \& JMP Predictor

Where,

- $\mathrm{U}$ is the universe of all attributes, $\mathrm{A}$ are the conditional attributes \& $\mathrm{D}$ is the decision attribute

- $\mathrm{J}$ is the Jaccard Matrix

\subsection{JMP Flow Chart}

In this section the flow chart of the JMP Systematic Structure Stages flowchart Figure (1). 


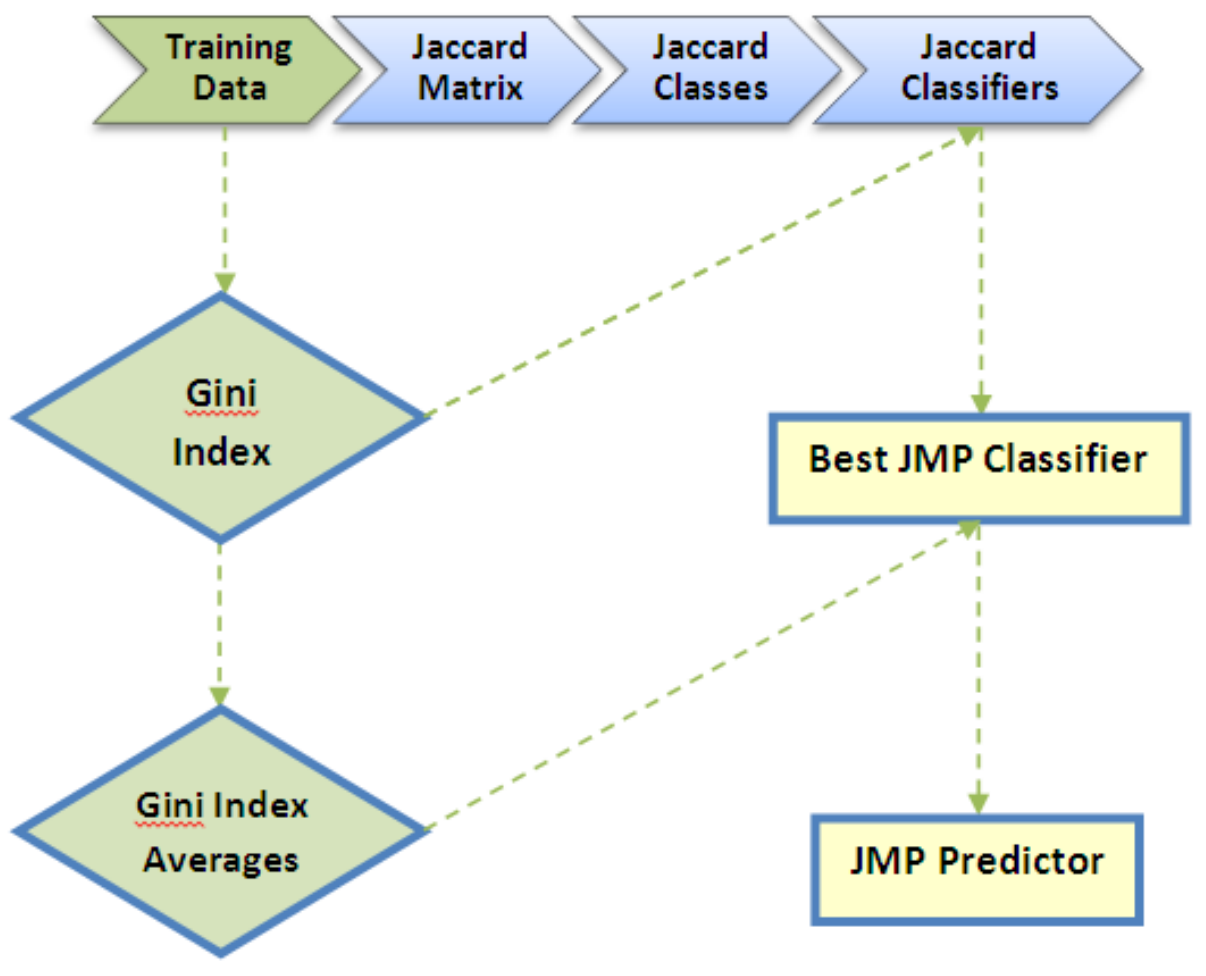

Figure (1): JMP Flow Chart

\subsection{Jaccard Matrix}

The $1^{\text {st }}$ stage in our JMP, Calculating Jaccard matrix of the Rheumatic Fever Data conditional attributes of Table (2).The results are given below in Table (3) Approximated to 2 decimals.

\begin{tabular}{|c|c|c|c|c|c|c|c|c|}
\hline & $\mathbf{S}$ & $\mathbf{F}$ & $\mathbf{A}$ & $\mathbf{R}$ & $\mathbf{K}$ & $\mathbf{E}$ & $\mathbf{P}$ & $\mathbf{H}$ \\
\hline $\mathbf{S}$ & 0.00 & 0.43 & 0.43 & 0.43 & 0.43 & 0.43 & 0.57 & 0.71 \\
\hline $\mathbf{F}$ & 0.43 & 0.00 & 0.00 & 0.29 & 0.57 & 0.57 & 1.00 & 0.57 \\
\hline $\mathbf{A}$ & 0.43 & 0.00 & 0.00 & 0.29 & 0.57 & 0.57 & 1.00 & 0.57 \\
\hline $\mathbf{R}$ & 0.43 & 0.29 & 0.29 & 0.00 & 0.57 & 0.57 & 0.71 & 0.57 \\
\hline $\mathbf{K}$ & 0.43 & 0.57 & 0.57 & 0.57 & 0.00 & 0.29 & 0.43 & 0.29 \\
\hline $\mathbf{E}$ & 0.43 & 0.57 & 0.57 & 0.57 & 0.29 & 0.00 & 0.43 & 0.29 \\
\hline $\mathbf{P}$ & 0.57 & 1.00 & 1.00 & 0.71 & 0.43 & 0.43 & 0.00 & 0.43 \\
\hline $\mathbf{H}$ & 0.71 & 0.57 & 0.57 & 0.57 & 0.29 & 0.29 & 0.43 & 0.00 \\
\hline
\end{tabular}

Table (3): Rheumatic Fever Conditional Attributes Jaccard Matrix

\subsection{Jaccard Classes}

The next stage in our classifier to divide the values of the Jaccard matrix of Table (3) into three classes; $(\mathrm{J}=0,0<\mathrm{J} \leq 0.5,0.5<\mathrm{J} \leq 1)$ and obtaining the conditional attributes classes introducing the output of this stage in Table (4) of the conditional attributes Jaccard classes. 


\begin{tabular}{|c|c|c|c|}
\hline & $\boldsymbol{J = 0}$ & $\mathbf{0}<\boldsymbol{J} \leq \boldsymbol{0 . 5}$ & $\mathbf{0 . 5}<\boldsymbol{J} \leq \boldsymbol{1}$ \\
\hline $\mathbf{S}$ & $\{S\}$ & $\{F, A, R, K, E\}$ & $\{P, H\}$ \\
\hline $\mathbf{F}$ & $\{F, A\}$ & $\{S, R\}$ & $\{K, E, P, H\}$ \\
\hline $\mathbf{A}$ & $\{F, A\}$ & $\{S, R\}$ & $\{K, E, P, H\}$ \\
\hline $\mathbf{R}$ & $\{R\}$ & $\{S, F, A\}$ & $\{K, E, P, H\}$ \\
\hline $\mathbf{K}$ & $\{K\}$ & $\{S, E, P, H\}$ & $\{F, A, R\}$ \\
\hline $\mathbf{E}$ & $\{E\}$ & $\{S, K, P, H\}$ & $\{F, A, R\}$ \\
\hline $\mathbf{P}$ & $\{P\}$ & $\{K, E, H\}$ & $\{S, F, A, R\}$ \\
\hline $\mathbf{H}$ & $\{H\}$ & $\{K, E, P\}$ & $\{S, F, A, R\}$ \\
\hline
\end{tabular}

Table (4): Conditional Attributes Jaccard Classes

\subsection{JMP Classifiers}

Comparing the conditional attributes row classes of Table (4) to get the Jaccard classifiers in Table (5).

\begin{tabular}{|c|c|c|}
\hline $\boldsymbol{J}=\mathbf{0}$ & $\mathbf{0}<\boldsymbol{J} \leq \mathbf{0 . 5}$ & $\mathbf{0 . 5}<\boldsymbol{J} \leq \boldsymbol{1}$ \\
\hline$\{S\}$ & $\{S\}$ & $\{S\}$ \\
\hline$\{F, A\}$ & $\{F, A\}$ & $\{F, A, R\}$ \\
\hline$\{R\}$ & $\{R\}$ & $\{K, E\}$ \\
\hline$\{K\}$ & $\{K\}$ & $\{P, H\}$ \\
\hline$\{E\}$ & $\{E\}$ & \\
\hline$\{P\}$ & $\{P\}$ & \\
\hline$\{H\}$ & $\{H\}$ & \\
\hline
\end{tabular}

Table (5): Conditional Attributes Jaccard Classifier

One can note that the mining Jaccard classifier is, $0.5<\mathrm{J} \leq 1$ classifier which has the least number of sets and the division of the attribute sets are very appropriate with the medical sight. Deciding which the best classifier is depends on the following calculations.

\subsection{Rheumatic Fever Data Gini Index}

Applying the Gini index rules in Section (2.3) on the data in Table (2), the values of the Gini Index of each attribute is calculated and the results are summarized and approximated to two decimal spaces in Table (6) and Chart (1) using the frequencies of all attributes which are represented in Figure (2) respectively. 


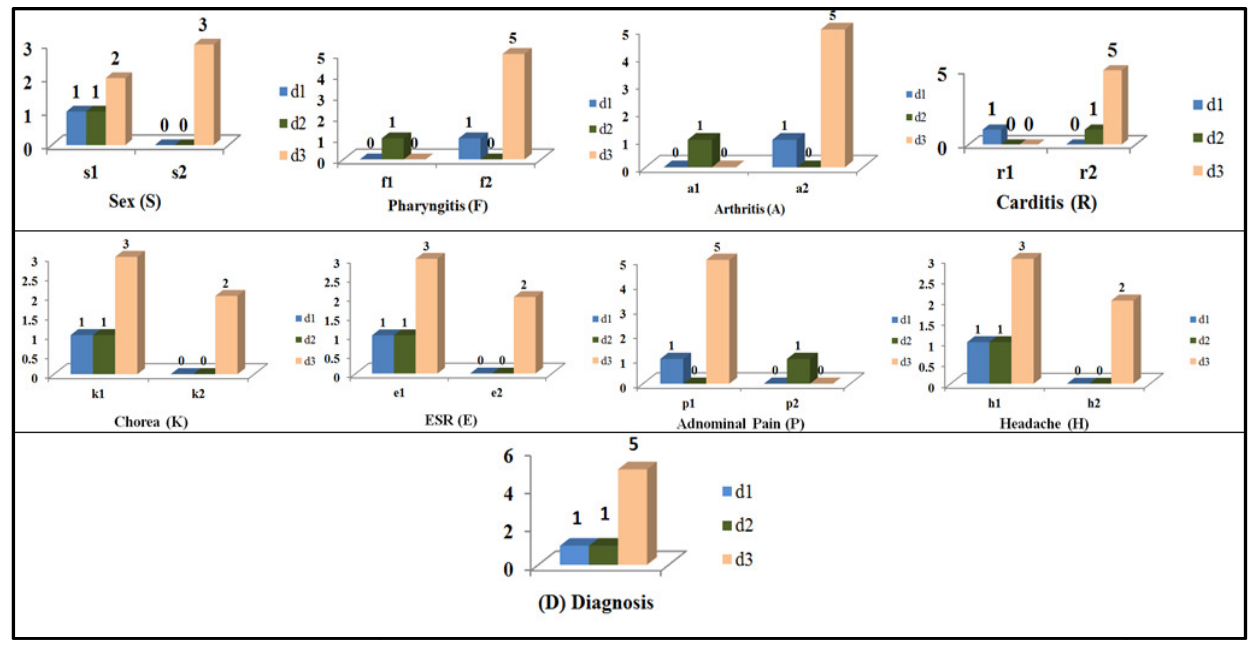

Figure (2): Rheumatic Fever Data Frequencies

\begin{tabular}{|c|c|}
\hline At. & Gini Index \\
\hline S & 0.35 \\
\hline F & 0.24 \\
\hline A & 0.24 \\
\hline R & 0.24 \\
\hline K & 0.4 \\
\hline E & 0.4 \\
\hline P & 0.24 \\
\hline H & 0.4 \\
\hline D & $\mathbf{0 . 4 5}$ \\
\hline
\end{tabular}

Table (6): Rheumatic Fever Gini Index

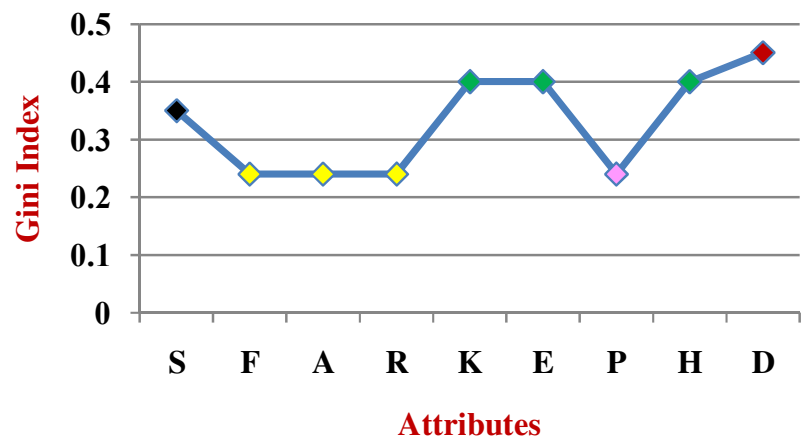

Chart (1): Rheumatic Fever Attributes Gini Index 


\subsection{Best JMP Classifier}

Deciding the best Jaccard classifier of Table (5) depends on the conditional attributes Gini Index of Table (6). So, the JMP classifier will be $0.5<\mathrm{J} \leq 1$ the best JMP classifier as in Table (7).

\begin{tabular}{|c|}
\hline $0.5<J \leq 1$ \\
\hline$\{S\}$ \\
\hline$\{F, A, R\}$ \\
\hline$\{K, E\}$ \\
\hline$\{P, H\}$ \\
\hline
\end{tabular}

Table (7): Best JMP Classifier

\subsection{JMP Predictor}

This stage to determine which set of the best JMP classifier sets to be the JMP predictor which we can use it with any prediction technique for predicting the diagnosis for any test data, by calculating the Gini index averges for each set of the best JMP classifier of Table (7) represented in Table (8) and Chart (2).

\begin{tabular}{|c|c|}
\hline $\mathbf{0 . 5}<\boldsymbol{J} \leq \mathbf{1}$ & Gini Average \\
\hline$\{S\}$ & 0.35 \\
\hline$\{F, A, R\}$ & 0.24 \\
\hline$\{K, E\}$ & 0.4 \\
\hline$\{P, H\}$ & 0.3 \\
\hline $\mathbf{D}$ & $\mathbf{0 . 4 5}$ \\
\hline
\end{tabular}

Table (8): Best JMP Classifier Gini Averages

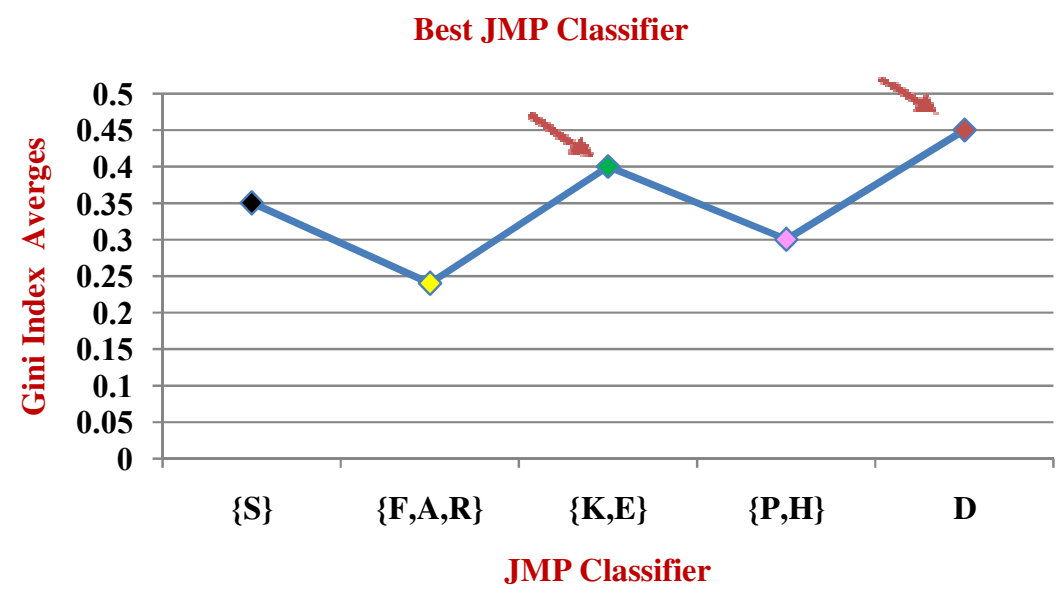

Chart (2): Best JMP Classifier Gini Averages 
From Chart (2) we can find that the value of the Gini average of $\{\mathrm{K}, \mathrm{E}\}$ is the closest value to the diagnosis Gini and this indicates that $\{\mathrm{K}, \mathrm{E}\}$ will be the JMP predictor attributes.

\author{
JMP Predictor $=\{K, E\}$
}

\title{
4.CONCLUSION
}

A new Statistical Data Mining (SDM) technique is initiated in this paper using Jaccard Mining Procedure (JMP) contributing a novel classifier \& predictor depending on Jaccard (J) distance matrix and Gini Index Measure. Applying JMP on real life application of Rheumatic Fever data diagnosis to see the accuracy of its applicability and the result was very accurate for the diagnosis of the data specialist. JMP opens the way for other new SDM techniques using an alternative distance measures and other accuracy measures according to the data type.

\section{REFERENCES}

[1] A.M. Kozae, M.M.E. Abd El-Monsef, and S. Abd El-Badie, New Approaches for Data Reduction in Generalized Multi-valued Decision Information System (GMDIS): Case Study of Rheumatic Fever Patients, The 2nd International Conference on MATHEMATICS: TRENDS AND DEVELOPMENTS, Egyptian Mathematical Society (ETMS), December 27-30, 232-BM, 2007, Cairo - EGYPT.

[2] Chun LT, Reddy DV, Yamamoto LG. Rheumatic fever in children and adolescents in Hawaii. Pediatrics. 1987; 79: 549-552.

[3] Han J. and Kamber M. Data Mining: Concepts and Technique, 2nd ed., the Morgan Kaufmann Series in Data Management Systems, Jim Gray, Series Editor Morgan Kaufmann Publishers, 2006. ISBN 155860-901-6.

[4] M.E. Abd El-Monsef, E. A. Rady, A. M. Kozea, W. A. Hassanein and S. Abd El-Badie What is the Major Power Linking Statistics \& Data Mining?, International Journal of Data Mining \& Knowledge Management Process , 2013, Volume 3, Number 6, ISSN 2230-9608, AIRCC Co.

[5] Oracle, Database Online Documentation Library Master Index, Copyright (C) 2014, Oracle and/or its affiliates. All rights reserved. http://docs.oracle.com/cd/E11882_01/nav/mindx.html

[6] Paul E. Black, "algorithm", in Dictionary of Algorithms and Data Structures [online], Vreda Pieterse and Paul E. Black, eds. 10 January 2007. (accessed TODAY) Available from: http://www.nist.gov/dads/HTML/algorithm.html

[7] Thair Nu Phyu, Survey of Classification Techniques in Data Mining, International Multi Conference of Engineers and Computer Scientists (IMECS), 2009, Hong Kong, Vol I. 


\section{Authors}

Mohamed Ezzat Abd El- Monsef, Professor of Pure Mathematics, Mathematics Department, Faculty of Science, Tanta university, Egypt. He was the former dean of the faculty. He is a member in many mathematical societies. He got the membership of the National Committee for Mathematics. He is a supervisor on many MS. C. and Ph. D. Thesis in many universities in and out of Egypt. He is a member in, ERS Group, http://www.cba.edu.kw/abo/rough-sets-working-group.html and the Egyptian Mathematical Society http://etms-eg.org Homepage: http://at.yorku.ca/h/a/a/a/48.html

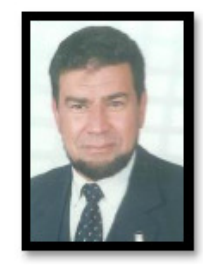

El-Houssainy Abd El-Bar Rady, Professor of Applied Statistics Applied Statistics Department, I.S.S.R, Cairo, Egypt. His Ph.D. in Statistics, from Oregon State University (OSU), USA. He was the Director of the Statistical and Econometrical Consultation Center. He got many awards, Sarhan Award of the Scientific research Academy, Egypt, Lee Award, OSU, USA, Member of the Honor Society of Phi Kappa Phi, USA and Thabet El-Sherief Award, ISSR, Cairo University.

Homepage: http://issr.cu.edu.eg/web_people_faculty_det.aspx?fac_id=31

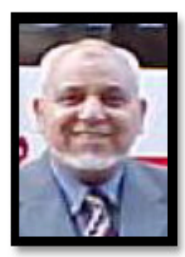

Abd El-Monem Mohamed Kozae, Professor of Pure Mathematics, Mathematics Department, Faculty of Science, Tanta university, Egypt He is a supervisor on many MS. in $\mathrm{C}$. and $\mathrm{Ph}$. D. Thesis in and many universities out of Egypt. He is a member in many mathematical societies, ERS Group, http://www.cba.edu.kw/abo/rough-sets-workinggroup.html, IRSS http://roughsets.home.pl/www/ and Egyptian Mathematical Societyhttp://etmseg.org Homepage:http://telc.tanta.edu.eg/hosting/pro6/pro6_index.html

Wafaa Anwar Abd El-Latif, Lecturer of Mathematical Statistics, Mathematics Department, Faculty of Science, Tanta University, Egypt. She got her BS. C., 2000, by excellence with honors degree, Her Ms. C. degree, 2004. And she got her Ph.D. degree, 2007, Titled "Uncertainty in Statistics", 2007, Faculty of Science, Tanta University, Egypt. She is a member in, ERS Group, http://www.cba.edu.kw/abo/rough-sets-workinggroup.html and Egyptian Mathematical Society http://etms-eg.org Homepage: http://tdb.tanta.edu.eg/staff_data/Staff\%20Detailed\%20Data-ar.aspx?MemberID=847
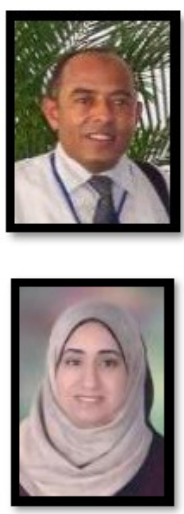

Soaad Abd El-Badie Attia El-Afefy, Ph. D. Student, Mathematics Department, Tanta University, Egypt. She got her Ms. C. Titled "A New Data Reduction Approach", 2006, Faculty of Science, Tanta University, Egypt. She got the Best Student Presentation and Best Student Paper Awards. She worked as teaching assistant at GUC, She is a member in, ERS Group http://www.cba.edu.kw/abo/rough-sets-working-group.html,IRSS http://roughsets.home.pl/www/, and Egyptian Mathematical Society http://etms-eg.org Homepage: http://www.savvymore.mysite.com

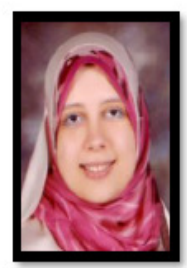

